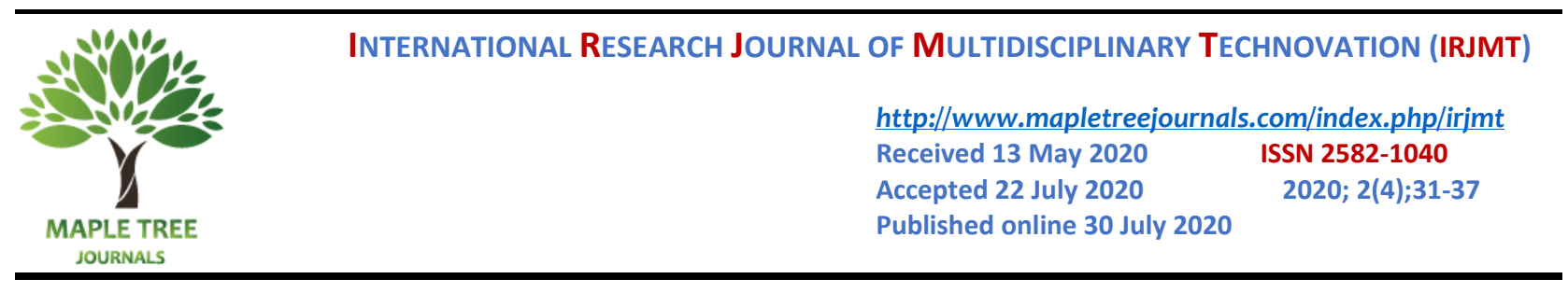

\title{
Smart Voice Assistant for Library System
}

\author{
S. Nirmal Kumar ${ }^{1, *}$, K. Prathinan ${ }^{1}$, G. Suresh ${ }^{1}$, P. Prema ${ }^{1}$ \\ ${ }^{1}$ Department of Computer Science and Engineering, Dhana Lakshmi College of Engineering, Chennai - 601301, \\ Tamil Nadu, India.
}

*Corresponding author E-Mail ID: snkrock106@gmail.com

DOI: https://doi.org/10.34256/irjmt2045

\begin{abstract}
Library plays an important role in student's life. It is also helpful for the research scholars. Today libraries are established in all places. Finding a book in the library is a herculean task. Some people even loose interest while searching the required book. Searching for books in computer also takes much time. In our project we use voice recognition to find books which will make the herculean task easier. Even the people without system knowledge can access this and find books in an easier way. It reduces the time in searching books and makes the library user friendly.
\end{abstract}

Keywords: IoT, Raspberry pi 3 model b, Speech recognition, Speech to text process.

\section{Introduction}

Library is a huge collection of books. The proper arrangement of books in given collection of order that makes it simple for the user to locate a specific book. This process used in large libraries having a huge collection [1-3]. They are not efficient and managed manually. The time has been wasted by searching for the book in the library. This problem mostly occurs in libraries, where number books is enormous. Thus, the fact of all books is arranged in classifications, the area of classification should first be known to the user becomes more acquainted with certain specific class of books is placed. In such a condition there should be a need through client can definitely find the area of any book by simply saying its name.

In this proposed system we use a microphone and speaker to establish interactive communication between the user and the system [4]. With the speech recognition library, we can process the user's query and respond by providing the requested information to the user. Speech recognition is the ability to receive and interpret dictation to understand and carry out given commands [5]. Voice recognition has gained prominence and use with the rise of AI and intelligent assistant. Voice recognition systems enable user to interact with simply way of speaking [6].

This technology provides smart searching for finding books from the libraries in an efficient way rather than finding or searching the books manually as in the conventional or traditional methods. Searching books manually which will take more time to find a book. This device will help to search and find the book quickly and efficiently [7-9]. As this device is using voice recognition technology it helps in searching books very easier than the manual searching.

\section{A. Existing System}

In the libraries the books are searched and founded manually. People will search the books which they wanted specifically from catalogue which is produced by the library. 
After finding the book from the catalogue the need to note the book's location number or any other locating techniques of the libraries. So, by searching the locating number they could able to find the book. If the people can't able to find their book in the catalogue, they have to ask the librarian for getting the book [10].

\section{B. Literature Survey}

This paper addresses different configurations of two layer and three layers neural networks approach for the low resources like gujarati. ear microphone recorded up to $2.3 \mathrm{kHz}-4 \mathrm{kHz}$ Noise hum $-90 \mathrm{~dB}$ around $200 \mathrm{~Hz}$. MFCC and Real Cepstral Coefficient (RC) Conjugate Gradient (CG)Algorithm and Levenberg-Merquardt (LM) Algorithm are used. This can be accomplished by discourse acknowledgment framework, which allows an information processor to distinguish the words an individual expresses in a receiver or phone, and changes over them into written text Television broadcasts containing both male and female speaker recorded and 10 Tamil spoken digits (0-9) and 5 spoken names from 30 different speakers Sampling rate $16 \mathrm{kHz}$.

\section{Proposed System}

This system works on speech recognition technology (see figure 1). It uses raspberry pi instead of using costly computers. This process will convey the message through USB speaker. It is used as an individual device for libraries. This process will replace the man work to system work and also reduce the time consumption. By using this concept user can find the books in easy way without any trouble and tension it won't reduce the User interest [11]. The location of the book will be guided through Speaker. By this way the user will find the book location easily.

\section{A. Materials and Methods}

USB speakers \& microphones: It doesn't require audio drivers used by other speaker systems; rather, they use the system's USB drivers. In effect, USB speakers and mic include a built-in sound card. This makes them an excellent replacement option for a malfunctioning or inoperative sound card (see figure 2 and figure 3 ).

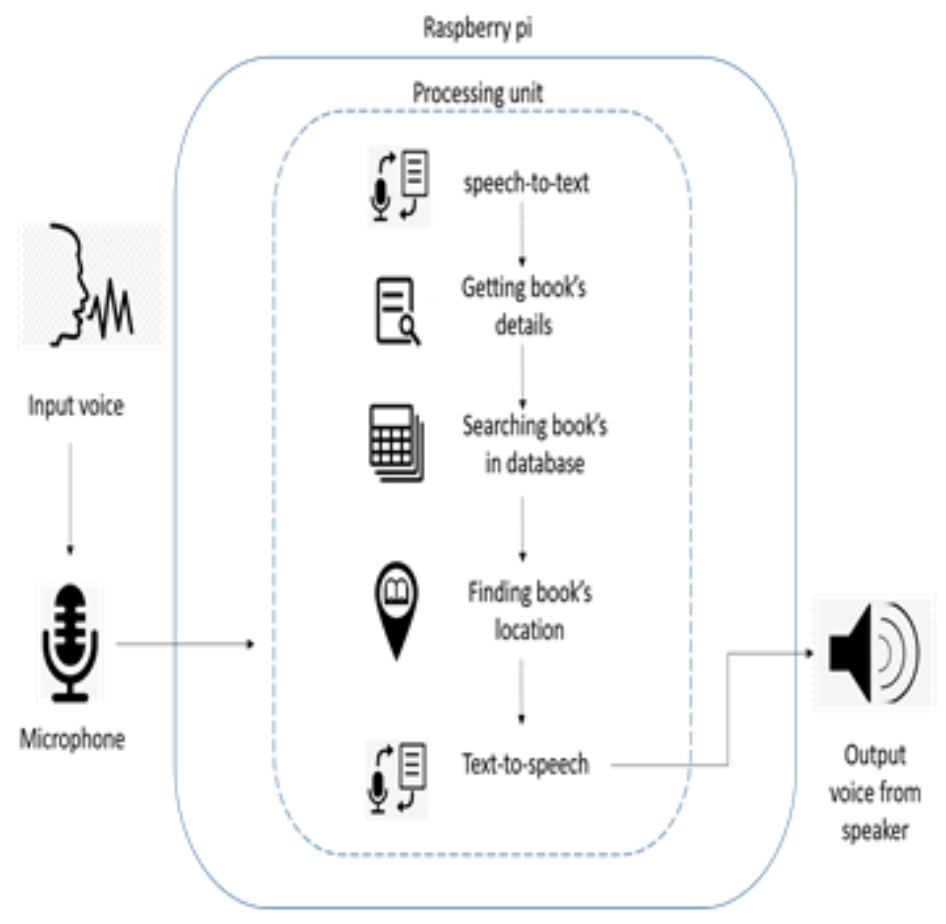

Fig 1. proposed system architecture 


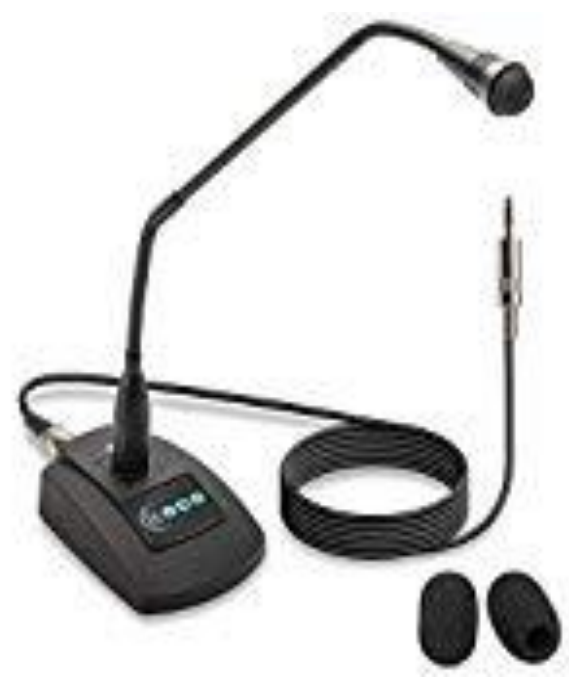

Fig 2. Microphone

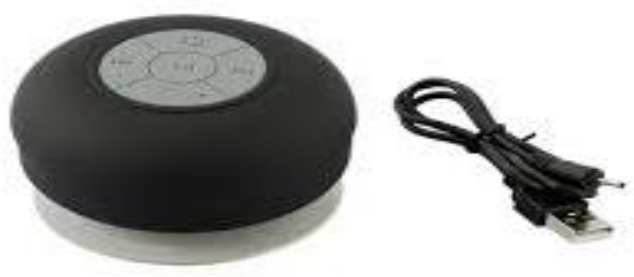

Fig 3. Speaker

1. Speech recognition: The first component of speech recognition is, of course, speech. Discourse must be changed over from physical sound to an electrical sign with a mouthpiece, and afterward to advanced information with a simple to-computerized converter (see figure 4).

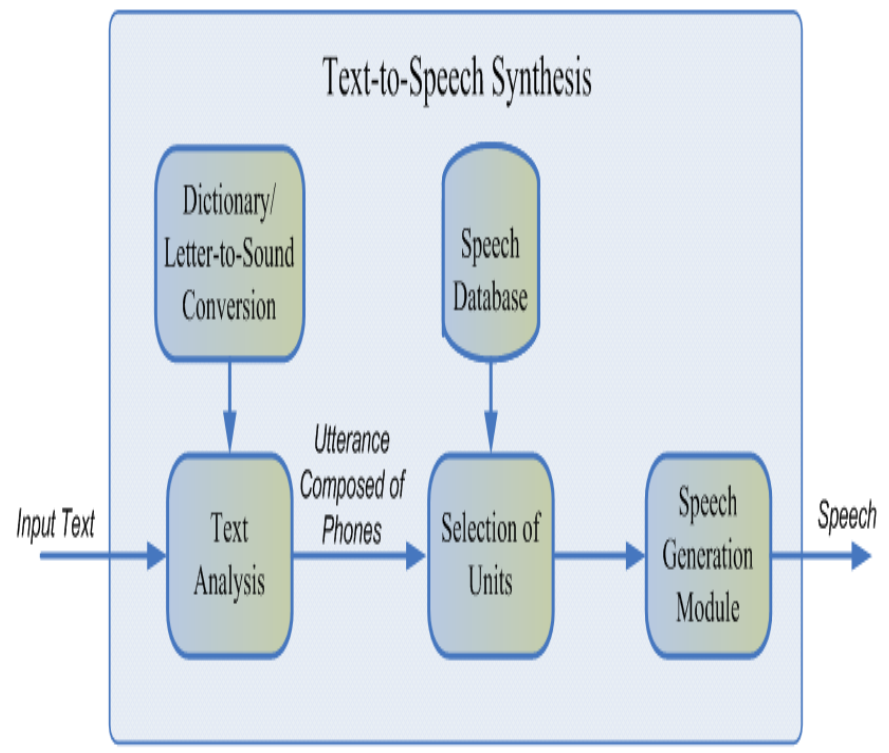

Fig 4. Text to speech 
Once digitized, a few models can be utilized to interpret the sound to content. To disentangle the discourse into content, gatherings of vectors are coordinated to at least one phoneme - a central unit of discourse. This figuring requires preparing, since the sound of a phoneme differs from speaker to speaker, and even fluctuates starting with one expression then onto the next by a similar speaker [12-15]. A unique calculation is then applied to decide the most probable word (or words) that produce the given grouping of phonemes.

2. Raspberry Pi 3: This board is a miniature marvel, packing considerable computing power into a footprint no larger than a credit card (see figure 5). It's prepared to do some astonishing things, yet there are a couple of things you're going to need to know before you dive straight into the thorn fix [16-17].

Since you have a fundamental comprehension of how the Pi varies from other figuring gadgets, it's an ideal opportunity to begin. If you've just received your Pi, take it out of its protective anti-static bag and place it on a flat, nonconductive surface before continuing with this chapter. This was proposed to be exceptionally reliant upon task stringing and guidance set use. Benchmarks showed the Raspberry Pi 3 to be approximately 80\% faster than the Raspberry Pi 2 in parallelized tasks. The Raspberry Pi Foundation uses Raspbian OS.

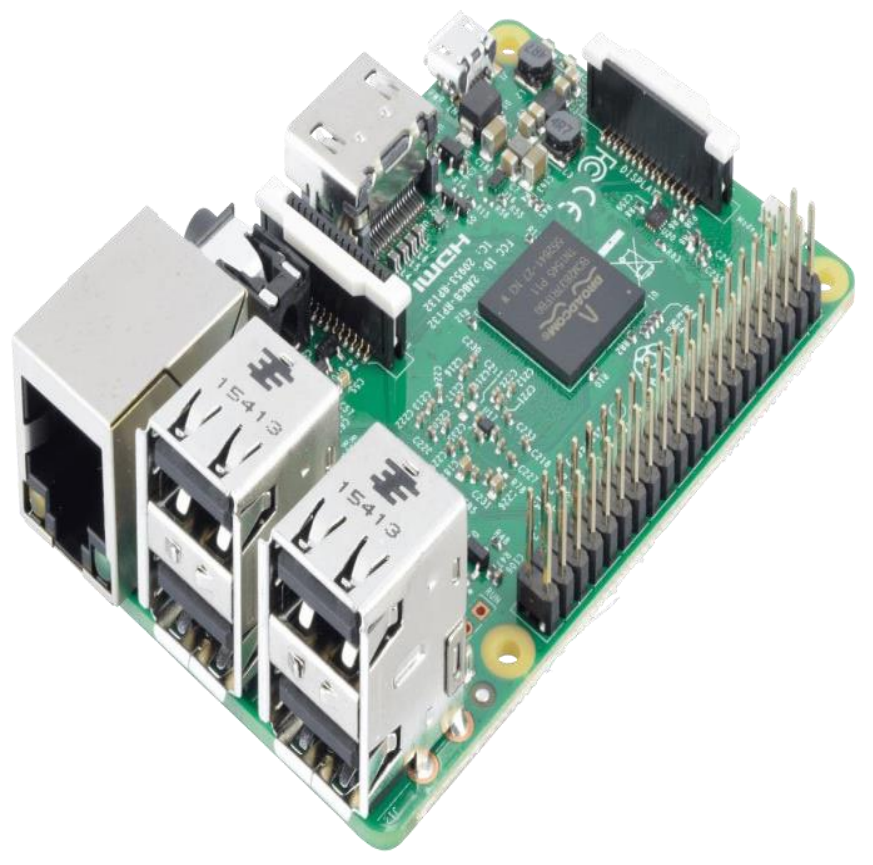

Fig 5. Raspberry Pi 3(MODEL B)

\section{System Design}

This system design consists of three units 1. Microphone (input unit) 2. Raspberry pi 3 (processing unit) 3. Speaker (output unit)

\section{A. Input Unit}

The person will convey the command in voice through microphone. It is easy to get identify the author of book name and section.

\section{B. Processing Unit}

In this processing unit is the raspberry pi. The raspberry pi is getting the input from the USB microphone. Speech must be converted from physical sound to an electrical signal with a 
microphone, and then to digital data with an analog-to-digital converter and it convey the output through the USB speaker.

\section{Output Unit}

After the processing unit, this unit will convey the command to speaker and its easily recognize by that person (see figure 5).

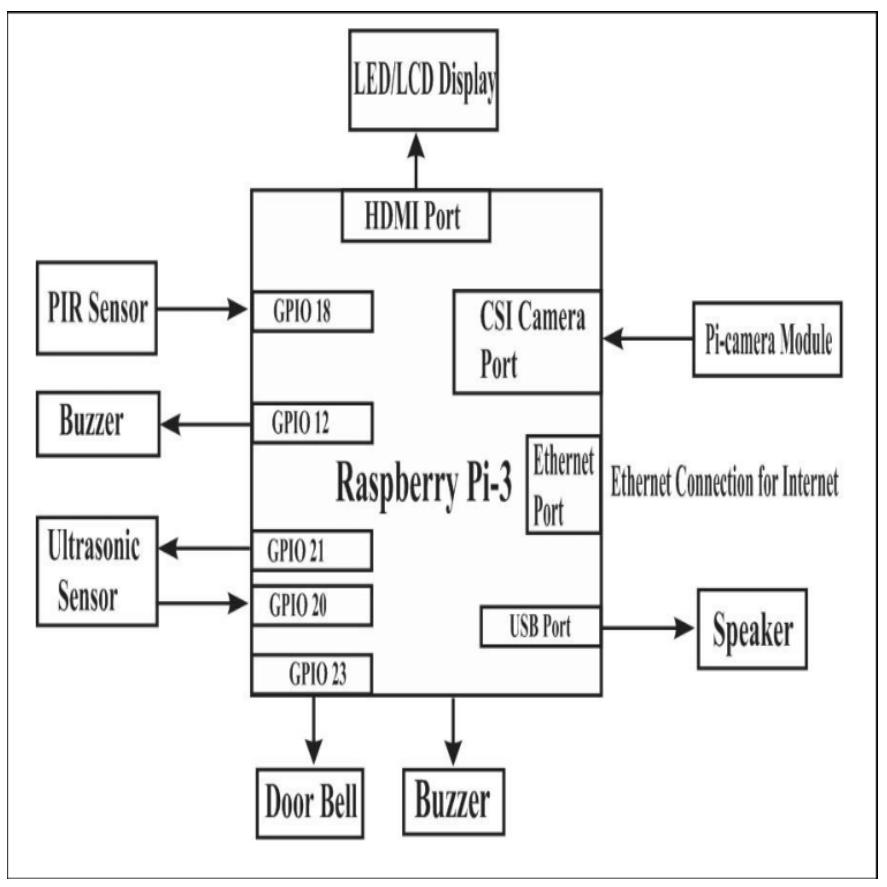

Fig 5. Block diagram

\section{Experiment and Result}

Thus, the raspberry pi help to interact with human for correct way of Speech recognition. The ability of a machine learning to identify words and phrases in spoken language and convert them to a machine-readable format (see figure 6). More sophisticated software has the ability to accept natural speech this way convey the location command to the user to identify the book.

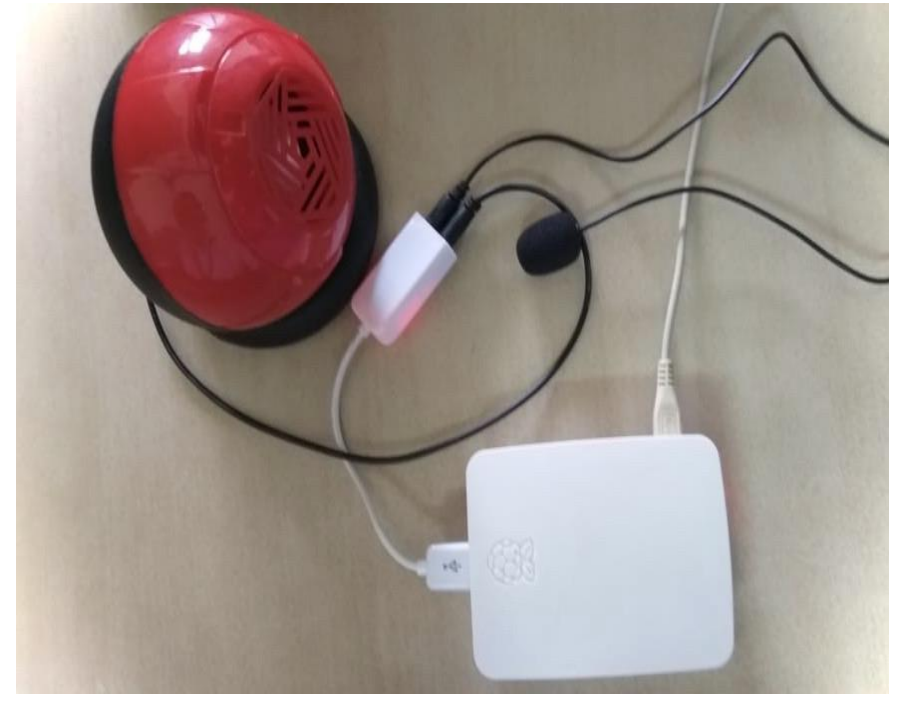

Fig 6. Output 


\section{Conclusion}

Thus, in this paper, the objective of the proposed system has been achieved. The vision of this project is to search books in libraries without any difficulties instead of searching the manually. Smart library management system is developed using a voice recognition technology which is very helpful for searching the books very effectively and it saves more time and reduces the difficulties for searching and finding the books. As the system is using raspberry pi it is very efficient and very convenient for accessing and working. Hence a new technology has been proposed it will be very for helpful for future upcoming generate.

\section{References}

[1] H. Kekre, A. A. Athawale, G. Sharma, (2011) Speech Recognition using Vector Quantization, In Proceedings of the International Conference \& Workshop on Emerging Trends in Technology, 400-403.

[2] Y. Pan, P. Shen, L. Shen, Speech Emotion Recognition using Support Vector Machine, International Journal of Smart Home, 6 (2012)101-108.

[3] K. M. Alhawiti, Advances in Artificial Intelligence Using Speech Recognition, World Academy of Science, Engineering and Technology, International Journal of Computer, Electrical, Automation, Control and Information Engineering, 9 (2015) 1397- 1354.

[4] Taabish Gulzar, Anand Singh, Dinesh Kumar Rajoriya, Najma Farooq, A Systematic Analysis of Automatic Speech Recognition: An Overview, International Journal of Current Engineering and Technology, 4 (2014) 1664-1675.

[5] A. Revathi, Y. Venkataramani, (2011) Speaker Independent Continuous Speech and Isolated Digit Recognition Using VQ and HMM, In 2011 International Conference on Communications and Signal Processing, IEEE, 198-202.

[6] A. Kuamr, M. Dua, T. Choudhary, (2014) Continuous Hindi Speech Recognition Using Gaussian Mixture HMM, In 2014 IEEE Students' Conference on Electrical, Electronics and Computer Science, IEEE, 1-5.

[7] D. Baby, J.F. Gemmeke, T. Virtanen, (2015) Exemplar-Based Speech Enhancement for Deep Neural Network Based Automatic Speech Recognition, In 2015 IEEE International Conference on Acoustics, Speech and Signal Processing (ICASSP), IEEE, 4485-4489

[8] Q.B. Nguyen, T.T. Vu, C.M. Luong, (2015) Improving Acoustic Model for English ASR System Using Deep Neural Network, In The 2015 IEEE RIVF International Conference on Computing \& Communication Technologies - Research, Innovation, and Vision for Future $(R I V F)$, IEEE, 25-29.

[9] S. Lee, Y. Lee, N. Cho, (2016) Multi-Stage Speech Enhancement for Automatic Speech Recognition, In 2016 IEEE International Conference on Consumer Electronics (ICCE), IEEE, 383-384

[10] Mohan, B. Jagan, (2014) Speech Recognition Using MFCC and DTW, In 2014 International Conference on Advances in Electrical Engineering (ICAEE), IEEE, 1-4.

[11] J. Arias, C. Busso, N. B. Yoma, Shape-Based Modeling of The Fundamental Frequency Contour for Emotion Detection in Speech, Computer Speech \& Language, 28 (2014) 278294.

[12] M. Abdelwahab, C. Busso, Domain Adversarial for Acoustic Emotion Recognition, IEEE/ACM IEEE Transactions on Audio, Speech and Language Processing, 26 (2018) 2423-2435.

[13] B. Schuller, F. Burkhardt, (2010) Learning with Synthesized Speech for Automatic Emotion Recognition, In 2010 IEEE International Conference on Acoustics, Speech and Signal Processing, IEEE, 5150-5153. 
[14] B. Schuller, Z. Zhang, F. Weninger, F. Burkhardt, Synthesized Speech for Model Training in Cross-Corpus Recognition of Human Emotion, International Journal of Speech Technology, 15 (2012) 313- 323.

[15] Luigi Atzori, Antonio Iera, Giacomo Morabito, The Internet of Things: A survey, Computer Networks, 54 (2010) 2787.

[16] Huang Shan, Voice Recognition Systems in The Telecom Prepaid Business Applications, Journal of Information Science, 2010.

[17] R. Solera Ureña, A.I. García-Moral, C. Peláez-Moreno, M. Martínez-Ramón, F. Díaz-dcMaría, Real-Time Robust Automatic Speech Recognition Using Compact Support Vector Machines, IEEE Transactions on Audio Speech and Language Processing, 20 (2012) 13471361.

\section{Acknowledgement Nil}

\section{Funding}

This study was not funded by any grant

\section{Conflict of interest}

None of the authors have any conflicts of interest to declare.

\section{About The License}

The text of this article is licensed under a Creative Commons Attribution 4.0 International License

\section{Cite this Article}

S. Nirmal Kumar, K. Prathinan, G. Suresh, P. Prema, Smart Voice Assistant for Library System, International Research Journal of Multidisciplinary Technovation, Vol 2, Iss 4 (2020) 31-37.

DOI: https://doi.org/10.34256/irimt2045 